\title{
ARTICLE
}

\section{Prognostic significance of esterase gene expression in multiple myeloma}

\author{
Romika Kumari ${ }^{1,2,3}$, Muntasir Mamun Majumder ${ }^{1,2}$, Juha Lievonen ${ }^{4}$, Raija Silvennoinen ${ }^{4}$, Pekka Anttila ${ }^{4}$, Nina N. Nupponen ${ }^{5}$, \\ Fredrik Lehmann ${ }^{5}$ and Caroline A. Heckman (iD) $1,2,3$
}

BACKGROUND: Esterase enzymes differ in substrate specificity and biological function and may display dysregulated expression in cancer. This study evaluated the biological significance of esterase expression in multiple myeloma (MM).

METHODS: For gene expression profiling and evaluation of genomic variants in the Institute for Molecular Medicine Finland (FIMM) cohort, bone marrow aspirates were obtained from patients with newly diagnosed MM (NDMM) or relapsed/refractory MM (RRMM). CD138+ plasma cells were enriched and used for RNA sequencing and analysis, and to evaluate genomic variation. The Multiple Myeloma Research Foundation (MMRF) Relating Clinical Outcomes in MM to Personal Assessment of Genetic Profile (CoMMpass) dataset was used for validation of the findings from FIMM.

RESULTS: MM patients (NDMM, $n=56$; RRMM, $n=78$ ) provided 171 bone marrow aspirates (NDMM, $n=56 ; \mathrm{RRMM}, n=115$ ). Specific esterases exhibited relatively high or low expression in MM, and expression of specific esterases (UCHL5, SIAE, ESD, PAFAH1B3, PNPLA4 and PON1) was significantly altered on progression from NDMM to RRMM. High expression of OVCA2, PAFAH1B3, $S I A E$ and USP4, and low expression of PCED1B, were identified as poor prognostic markers $(P<0.05)$. The MMRF CoMMpass dataset provided validation that higher expression of $P A F A H 1 B 3$ and SIAE, and lower expression of $P C E D 1 B$, were associated with poor prognosis.

CONCLUSIONS: Esterase gene expression levels change as patients progress from NDMM to RRMM. High expression of OVCA2, $P A F A H 1 B 3$, USP4 and SIAE, and low expression of PCED1B, are poor prognostic markers in MM, suggesting a role for these esterases in myeloma biology.

British Journal of Cancer (2021) 124:1428-1436; https://doi.org/10.1038/s41416-020-01237-1

\section{BACKGROUND}

Both antibody-drug conjugates (ADCs) and peptide-drug conjugates (PDCs) represent important therapeutic classes that enable the selective introduction of cytotoxic drugs into cancer cells over healthy cells, potentially improving efficacy and reducing systemic toxicity compared with non-conjugated versions of the same drug. ${ }^{1,2}$ Conceptual similarities between ADCs and PDCs include selective targeting and subsequent cellular internalisation, although the exact mechanism of action is specific to each individual conjugate. To release cytotoxic payloads within the target cell, ADCs are typically endocytosed and cleaved within the consequent lysosomal structure, whereas PDCs undergo hydrolytic cleavage in the cell via interactions with cell surface receptors, or after direct entry into the cytosol through the cell membrane. ${ }^{1-3}$ Various different enzyme classes may be involved in conjugate metabolism and release of the cytotoxic payload in $A D C s$ and PDCs, and hence research interest in metabolising enzymes is growing for both therapeutic approaches. ${ }^{1-3}$

The esterase enzyme family is a subclass of the hydrolase enzyme superfamily that functions to hydrolyse ester bonds. ${ }^{4,5}$ Many different esterases have been identified, which differ in their substrate specificity and biological function, and the expression of specific esterases may be dysregulated in cancer. Hydrolysing enzymes can be highly expressed in cancer cells, and have previously been implicated in the reprogramming of metabolic pathways, promotion of cancer pathogenesis, drug metabolism and drug toxicity. ${ }^{4,5}$ Esterases expressed in tumour cells may also differ in their stereoselectivity for hydrolysis of chiral esters compared with esterases expressed in healthy tissues. ${ }^{6-9}$ Esterases can themselves also be administered to treat haematological malignancies; for example, the enzyme asparaginase has been used as an effective agent to treat acute lymphoblastic leukaemia for many years, ${ }^{10}$ and it is also being investigated for the treatment of acute myeloid leukaemia. ${ }^{11}$

Esterase hydrolysis, therefore, represents an interesting potential strategy for selective activation of anticancer drugs within cancer cells that overexpress esterases, or which express esterases with different specificities, while minimising toxic effects on healthy cells and tissues. ${ }^{6}$ The study of esterase expression in cancer is still in its infancy, but interest is growing as a result of the development of novel PDCs such as melflufen (melphalan flufenamide), which utilises intracellular aminopeptidases and

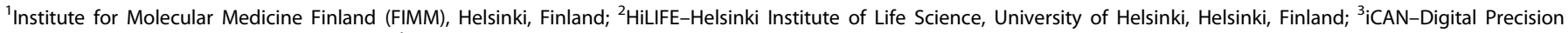

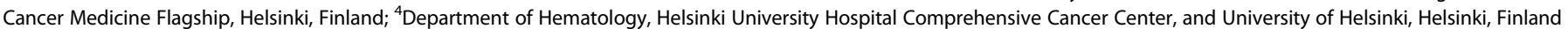
and ${ }^{5}$ Oncopeptides AB, Stockholm, Sweden

Correspondence: Caroline A. Heckman (caroline.heckman@helsinki.fi)

Received: 14 July 2020 Revised: 4 December 2020 Accepted: 11 December 2020

Published online: 3 February 2021 
esterases to release a cytotoxic payload in multiple myeloma cells. $^{3,12}$

Several esterases have previously been reported to be dysregulated in specific cancers, with some also having the potential to be predictive or prognostic biomarkers. For example, low expression of neurexophilin and PC-esterase domain family member 4 (NXPE4) mRNA is a prognostic marker for shorter survival in patients with colorectal cancer, ${ }^{13}$ and expression of platelet-activating factor acetylhydrolase 1B2 (PAFAH1B2) is inversely correlated with patient survival in pancreatic ductal adenocarcinoma. $^{14}$ In lung cancer, acetylcholinesterase (ACHE) may act as a tumour suppressor and is downregulated, ${ }^{15}$ and high expression of granzyme $A(G Z M A)$ is significantly associated with an improved prognosis in multiple cancer types. ${ }^{16}$ Sequencing studies have identified a number of genes that are mutated with high frequency in multiple myeloma, ${ }^{17,18}$ although genes encoding esterases do not feature prominently among them. This is most likely because esterase genes do not have high-frequency mutation rates in multiple myeloma, which in turn suggests that regulation of esterase gene expression occurs through mechanisms other than mutations. Still, expression of esterase genes can be dysregulated in this disease: carboxylesterase 1 (CES1), CES2 and butyrylcholinesterase $(B C H E)$ can both be overexpressed in multiple myeloma cells, ${ }^{19,20}$ and $B C H E$ is associated with a poor prognosis in multiple myeloma and dichotomously expressed, suggesting its tightly controlled regulation. ${ }^{21}$ Also, expression of ubiquitin carboxyl-terminal esterase L1 (UCHL1) in samples from patients with newly diagnosed multiple myeloma (NDMM) correlated with a high-risk subgroup of myeloma. ${ }^{22}$ Other studies have suggested possible mechanisms for the role of esterases in the pathogenesis of multiple myeloma; these include impaired oxidative/antioxidative balance due to reduced activity of serum paraoxonase- 1 and arylesterase, ${ }^{23}$ and inhibition of ubiquitinspecific protease 14 and ubiquitin-C-terminal hydrolase-5 (UCHL5). ${ }^{24,25}$

In this study, we evaluated the biological significance of a panel of esterases in multiple myeloma using gene expression profiling, cytogenetic profiling and clinical outcome analyses. We demonstrate that specific esterases exhibit relatively high or low expression in multiple myeloma, that the esterase expression profile changes on the progression of the disease, and that high or low expression of individual esterases is associated with poor prognosis. Although single-nucleotide variants (SNVs) were rare in the esterase genes, several had duplication and deletion copy number alterations.

\section{METHODS}

Sample collection and plasma cell enrichment

For gene expression profiling and evaluation of genomic variants in the Institute for Molecular Medicine Finland (FIMM) cohort, bone marrow aspirates were obtained from multiple myeloma patients after obtaining written informed consent and following protocols approved by an ethical committee of the Helsinki University Hospital Comprehensive Cancer Center, and in compliance with the Declaration of Helsinki. Matched patient skin biopsies were collected (also with informed consent) at the same time and from the same site as bone marrow aspirates, and in accordance with approved protocols, for constitutional DNA analysis. Bone marrow mononuclear cells were isolated by FicollPaque gradient centrifugation (GE Healthcare), and CD138+ plasma cells enriched by immuno-magnetic bead selection (StemCell Technologies).

RNA sequencing and analysis

RNA was extracted from CD138+ plasma cells using the AllPrep ${ }^{\oplus}$ DNA/RNA/miRNA Universal or miRNeasy kits (Qiagen). RNA integrity was measured on an Agilent Bioanalyzer 2100 instrument; only samples with RNA integrity $\geq 7$ were used for sequencing. Illumina-compatible RNA sequencing libraries were prepared using Scriptseq ${ }^{\mathrm{TM}}$ or Nextera technology and sequenced on Illumina HiSeq 1500 or 2500 instruments (Illumina). After preprocessing, filtered reads were aligned to the GRCh38 human reference genome using the STAR aligner tool. ${ }^{26}$ Gene read counts were normalised using the Reads Per Kilobase of transcript per million mapped reads (RPKM) method. In total, 51 annotated esterase genes (Supplementary Table S1) were identified in the human genome (assembly GRCh38) utilising the Ensembl release $99^{27}$ and $\mathrm{NCBI}^{28}$ databases, using the search term 'esterase' and further confirming the molecular function (gene ontology) of identified genes. A cut-off value of $>1$ RPKM was used to filter expressed esterase genes. DEseq $2^{29}$ was used to identify variation in gene expression in newly diagnosed versus relapsed/refractory samples. The contribution of esterase gene expression to survival outcome was estimated by Kaplan-Meier analysis and performed using expression-based filtering. For each esterase gene, samples were stratified into 'high' ( $\geq$ median expression) and 'low' (<median expression) expression groups. The significance of the difference between the two groups (high versus low expression) was deduced using a Mantel-Cox log-rank test.

\section{Exome sequencing and cytogenetics}

The DNeasy Blood \& Tissue kit or AllPrep DNA/RNA/miRNA Universal kit (Qiagen) was used to isolate genomic DNA from skin biopsies and CD138+ cells. The SeqCap EZ MedExome kit (Roche NimbleGen), SureSelect Clinical Research Exome kit or SureSelect Human All Exon V5 kit (Agilent Technologies) was used for exome capture. Sequencing was performed on HiSeq 1500 or 2500 instruments. VarScan2 somatic algorithm ${ }^{30}$ was implemented for calling somatic mutations, and mutation annotations were performed using SnpEff $4.04^{31}$ as described previously. ${ }^{32}$ Gene copy number variants (CNVs) were identified using the CopyCat tool (https://github.com/chrisamiller/copycat). Cytogenetics data were generated using fluorescence in situ hybridisation technology as described previously, ${ }^{33}$ following European Myeloma Network 2012 guidelines. $^{34}$

\section{Data validation}

To validate our results, clinical, gene expression and genomic variant data (somatic mutation and CNVs) were obtained from the Multiple Myeloma Research Foundation (MMRF) Relating Clinical Outcomes in MM to Personal Assessment of Genetic Profile (CoMMpass) study (https://research.themmrf.org, www.themmrf. org). The MMRF CoMMpass gene expression dataset includes 892 samples: $87 \%$ baseline/diagnosis, $12 \%$ progressive disease and $1 \%$ missing. See Supplementary Material for further details.

\section{RESULTS}

Patient population

In total, 134 patients provided 171 bone marrow aspirates for the FIMM dataset: 56 samples for the NDMM subgroup and 115 samples for the relapsed/refractory multiple myeloma (RRMM) subgroup (see Supplementary Fig. S1 for a patient/sample flow chart). Individual patients could provide samples only at diagnosis $(n=49)$, at both diagnosis and relapse $(n=7)$, only at first relapse $(n=59)$ or at recurring relapses, i.e., multiple relapse samples from one patient (second relapse, $n=13$; third relapse, $n=3$; fourth relapse, $n=2$; sixth relapse, $n=1$ ). The median age was similar in the NDMM and RRMM subgroups, and there was a higher proportion of males $(78 / 134,58.2 \%)$ in the total population (Table 1). More patients in the RRMM subgroup $(n=20,25.6 \%)$ had a 17 p deletion compared with the NDMM subgroup $(n=5$, $8.9 \%$; Fisher exact test $P=0.015$ ). Chromosome $1 \mathrm{q}$ gain was also more common in the RRMM subgroup $(n=44,56.4 \%)$ compared with the NDMM group $(n=14,25.0 \%$; Fisher exact test $P=$ 
Table 1. Patient characteristics, disease characteristics and prior therapies in patients with multiple myeloma in the in-house FIMM cohort.

Patient and disease characteristics by disease stage ${ }^{\mathrm{a}}$

\begin{tabular}{|c|c|c|c|}
\hline & $\begin{array}{l}\text { NDMM } \\
(n=56)\end{array}$ & $\begin{array}{l}\text { RRMM } \\
(n=78)\end{array}$ & $\begin{array}{l}\text { Total } \\
(N=134)\end{array}$ \\
\hline $\begin{array}{l}\text { Age at diagnosis, years, } \\
\text { median (range) }\end{array}$ & $64.5(26-84)$ & $63(41-81)$ & $64(26-84)$ \\
\hline Sex, female/male, $n$ & $26 / 30$ & $30 / 48$ & $56 / 78$ \\
\hline \multicolumn{4}{|l|}{ Cytogenetics, n (\%) } \\
\hline$t(11 ; 14)$ & $16(28.6)$ & $14(17.9)$ & $30(22.4)$ \\
\hline $\mathrm{t}(4 ; 14)$ & $7(12.5)$ & $18(23.1)$ & $25(18.7)$ \\
\hline$t(14 ; 16)$ & $1(1.8)$ & $2(2.6)$ & $3(2.2)$ \\
\hline$t(14 ; 20)$ & 0 & $2(2.6)$ & $2(1.5)$ \\
\hline $\operatorname{del}(17 p)$ & $5(8.9)$ & $20(25.6)$ & $25(18.7)$ \\
\hline $\operatorname{del}(13 q)$ & $36(64.3)$ & $40(51.3)$ & $76(56.7)$ \\
\hline 1q gain & $14(25.0)$ & $44(56.4)$ & $58(43.3)$ \\
\hline Missing & 0 & $2(2.6)$ & $2(1.5)$ \\
\hline \multicolumn{4}{|l|}{ ISS, $n(\%)$} \\
\hline 1 & $13(23.2)$ & $16(20.5)$ & $29(21.6)$ \\
\hline 2 & $26(46.4)$ & $22(28.2)$ & $48(35.8)$ \\
\hline 3 & 10 (17.9) & $16(20.5)$ & $26(19.4)$ \\
\hline Missing & $7(12.5)$ & $24(30.8)$ & $31(23.1)$ \\
\hline \multicolumn{4}{|c|}{ Treatment history of relapsed/refractory patients $(N=78)$} \\
\hline & $\begin{array}{l}\text { Exposed, } \\
\text { relapsed }\end{array}$ & $\begin{array}{l}\text { Exposed, } \\
\text { refractory }\end{array}$ & $\begin{array}{l}\text { Not } \\
\text { exposed }\end{array}$ \\
\hline \multicolumn{4}{|l|}{ Prior treatment, $n(\%)$} \\
\hline $\begin{array}{l}\text { Alkylating agents } \\
\text { (MEL, CPM) }\end{array}$ & $61(78.2)$ & $15(19.2)$ & $2(2.6)$ \\
\hline Bortezomib & $43(55.1)$ & $26(33.3)$ & $9(11.5)$ \\
\hline IMiDs & $29(37.2)$ & $33(42.3)$ & $16(20.5)$ \\
\hline
\end{tabular}

CPM cyclophosphamide, FIMM Institute for Molecular Medicine Finland, IMiD immunomodulatory imide drug, ISS International Staging System, MEL melphalan, NDMM newly diagnosed multiple myeloma, RRMM relapsed/ refractory multiple myeloma.

af a patient provided both NDMM and RRMM samples, this patient was included in the NDMM group. If a patient provided samples at multiple relapse stages and the diagnosis sample is missing, then data from the first relapse are included in the table.

0.0004). In the RRMM subgroup, previous treatments included alkylating agents in $97.4 \%$, bortezomib in $88.5 \%$ and immunomodulatory drugs in $79.5 \%$ of patients.

Esterase gene expression profile in multiple myeloma samples RNA extracted from 123 of 171 CD138+ plasma cell patient samples was suitable for RNA sequencing analysis, which included 41 samples from patients with NDMM, and 82 samples from patients with RRMM.

Esterase gene expression levels were ranked based on their abundance (Fig. 1a and Supplementary Table S1). The most abundant esterase mRNAs were ovarian tumour suppressor candidate 2 (OVCA2), PAFAH1B2, NXPE3, UCHL3, lipase A lysosomal acid type (LIPA), abhydrolase domain containing 10 (ABHD10), UCHL5, N-acetylneuraminate 9-0-acetyltransferase (CASD1), $A B H D 13$ and ubiquitin carboxyl-terminal hydrolase 4 (USP4), with a median log2[RPKM] range of 3.9 (OVCA2) to 2.1 (USP4). The least abundant esterases were asparaginase (ASPG), CES5A, arylacetamide deacetylase (AADAC), interleukin-17A (IL17A), neuroligin $4 \mathrm{Y}$ linked (NLGN4Y), paraoxonase $3(P O N 3)$, cholesterol esterase (CEL), $P O N 1$, neuroligin-2 (NLGN2) and NLGN1, with a median log2[RPKM] range of -10.7 (ASPG) to -5.1 (NLGN1; Fig. 1a). Similar esterase mRNA expression patterns were observed $(r=0.9, P$ value $=2.2 \mathrm{e}-$ 16) utilising the MMRF CoMMpass dataset $(N=892)$ (Supplementary Figs. S2, S3). In our FIMM dataset, the esterase genes clustered in four subgroups based on expression level, with group I having the highest and group IV having the lowest level of expression (Fig. 1b).

The mRNA expression profiles of several esterases were significantly different in samples from newly diagnosed patients versus those whose disease had relapsed or were refractory to treatment (Fig. 2). The expression levels of UCHL5, sialic acid acetyl esterase (SIAE), esterase D (ESD), PAFAH1B3 and PON1 were significantly higher $(P \leq 0.01$; adjusted $P<0.1)$ in RRMM versus NDMM samples, and the expression level of PNPLA4 was significantly lower $(P=0.007)$. In the MMRF CoMMpass dataset using paired samples (NDMM, $n=39$; RRMM, $n=45$ ), we observed similar expression patterns, with the median expression of the majority of these esterase genes being higher in RRMM samples. Whereas gene PNPLA4 had a conflicting expression pattern with expression being higher in RRMM samples in the CoMMpass dataset. However, none of the genes were found to have $P$ values $\leq 0.05$ (Supplementary Fig. S4) in the DEseq2 analysis. In addition, the expression of the esterase gene phospholipase A2 Group VII (PLA2G7; $P<0.001$ ) was significantly different in NDMM versus RRMM samples using paired samples from the MMRF CoMMpass data (data not shown).

DEseq2 analysis also revealed that out of all the esterases $(n=$ 6) predicted to be differentially regulated in NDMM versus RRMM samples in our dataset, only UCHL5 was upregulated in both RRMM samples as well as samples with 1q gain (Supplementary Fig. S5).

Prognostic significance of esterase expression in multiple myeloma

In our FIMM dataset, patient samples exhibiting high expression of OVCA2, PAFAH1B3, SIAE and USP4 were associated with a significantly poorer prognosis versus those with low expression (Fig. 3a, Supplementary Figs S6, S7 and Supplementary Table S2); median overall survival for high versus low expression samples was 68 versus 122 months for OVCA2 (hazard ratio (HR) 3.35, 95\% confidence limit (CL) 1.811-6.198; $P<0.0001), 73$ versus 122 months for PAFAH1B3 (HR 2.307, 95\% CL 1.31-4.062; $P=$ 0.0042), 68 versus 111 months for SIAE (HR 1.87, 95\% CL $1.083-3.226 ; P=0.027$ ) and 74 versus 122 months for USP4 (HR 1.76, $95 \% \mathrm{CL} 1.012-3.059 ; P=0.041)$. Genes with an overall expression of $<1$ RPKM were not included in this analysis. Expression of OVCA2 was significantly lower (Welch's $t$ test, $P=$ $0.003)$ in samples with del17p $(n=19)$ than in those without $(n=$ 104; Supplementary Fig. S8).

Patient samples exhibiting low expression of GZMA, PCED1B and NXPE3 were associated with poorer prognosis versus those with high expression (Fig. 3a, Supplementary Figs. S6, S7 and Supplementary Table S2); median overall survival for high versus low expression samples was 120 versus 55 months for GZMA (HR $0.517,95 \%$ CL 0.306-0.874; $P=0.013), 100$ versus 76 months for PCED1B (HR 0.546, 95\% CL 0.319-0.936; $P=0.026$ ) and 96 versus 76 months for NXPE3 (HR 0.578, 95\% CL 0.337-0.991). A visual comparison of the $P$ values for all data is shown (Supplementary Fig. S6).

The MMRF CoMMpass dataset provided validation that higher expression of PAFAH1B3 (HR 1.431; 95\% CL 1.054-1.943; $P=0.021$ ) and SIAE (HR 1.363;95\% CL 1.004-1.85; $P=0.046)$, and lower expression of PCED1B (HR 0.691; 95\% CL 0.51-0.934; $P=0.016$ ) were associated with poor prognosis (Fig. 3b). Several other esterases (NXPE4, UCHL5, PAFAH1B2, BPHL, NXPE1, UCHL3) not identified in the FIMM dataset were predicted to have a role in disease prognosis in the MMRF CoMMpass dataset (Supplementary Fig. S6). 

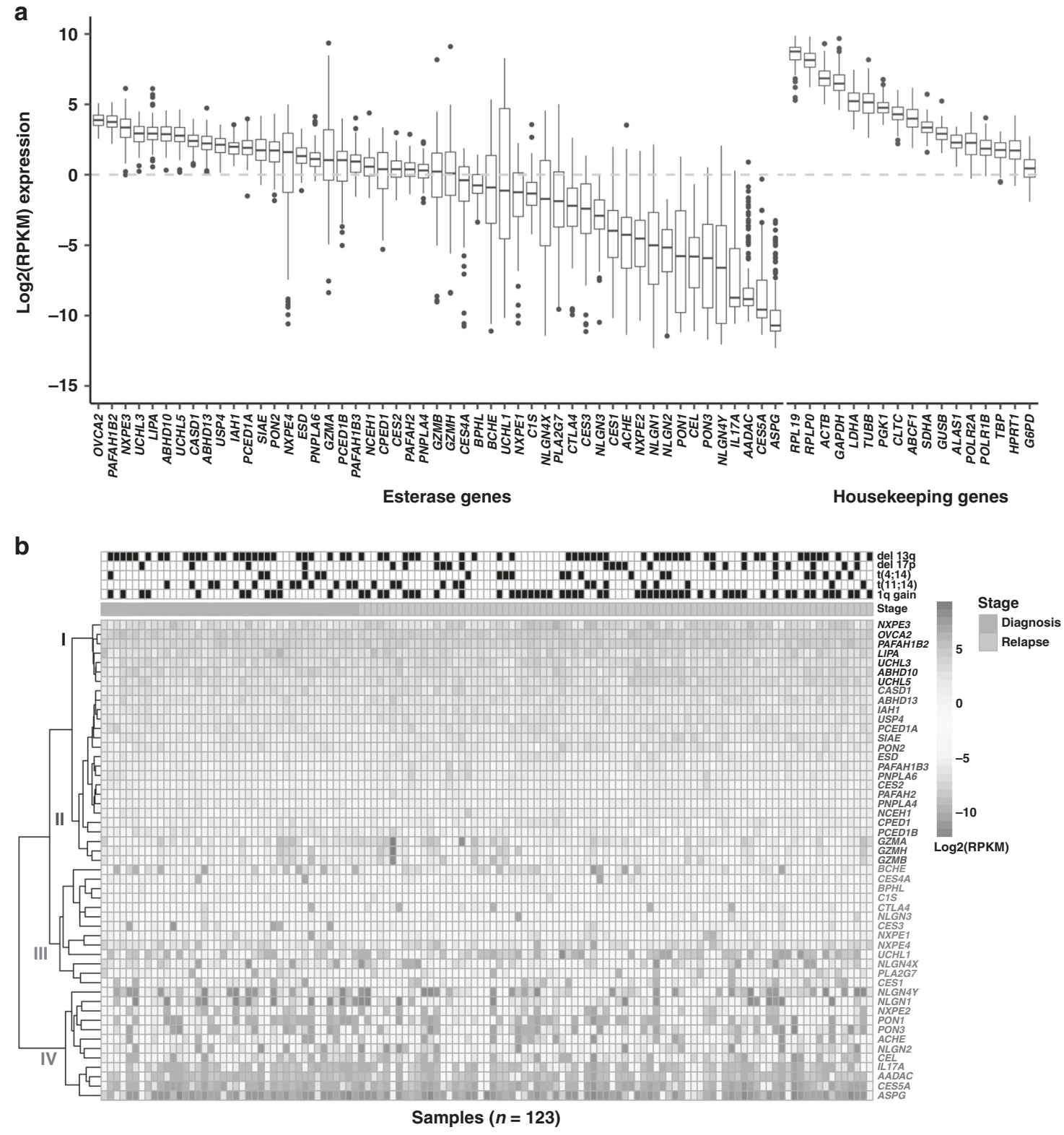

Fig. 1 Esterase gene expression profile in multiple myeloma samples from the in-house FIMM dataset. a Log2(RPKM) expression of esterases and housekeeping genes, ranked based on median expression values. ${ }^{a}$ b Esterase expression heatmap and hierarchical clustering based on expression level ${ }^{b}$. FIMM Institute for Molecular Medicine Finland, MM multiple myeloma, RPKM reads per kilobase of transcript per

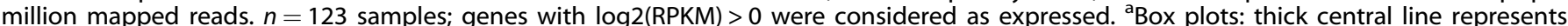
median; top and bottom lines of box represent third quartile and first quartile; whiskers indicate the variability in the data outside the upper and lower quartile; filled black circles represent outliers. ${ }^{b}$ The unsupervised hierarchical clustering of esterase gene expression profiles was performed using method complete-linkage and Manhattan distance measures.

Somatic mutation and copy number variation

Of 171 patient samples, 169 were successfully processed for exome sequencing, comprising 56 samples from patients with NDMM and 113 samples from patients with RRMM.

Exome sequencing data revealed that somatic SNVs are rare in esterase genes. In this cohort, somatic SNVs were observed in the CEL gene at a frequency of $3.5 \%$ (6/169 samples; no hotspot mutations, $n=6$ non-synonymous) and in the NXPE1 gene at a frequency of $2.3 \%$ (4/169 samples; no hotspot mutations, $n=4$ non-synonymous), while the frequency of SNVs in other esterase genes was below $2 \%$ (PCED1B 0.59\%, 1/169 samples; PAFAH1B3 0\%; SIAE 0\%; Supplementary Fig. S9). Mutation frequencies in esterase genes were also rare in the MMRF CoMMpass dataset, with all esterases being mutated in $<2 \%$ of samples ( $N=1164$; Supplementary Fig. S10).
In contrast, multiple esterase genes were found to have copy number alterations of both duplication and deletion types (Fig. 4). PNPLA6 was found to have a copy number gain in $30.8 \%(52 / 169)$ of samples, PAFAH1B3 had a copy number gain in $18.9 \%(32 / 169)$ of samples and CEL had a copy number gain in $17.2 \%(29 / 169)$ of samples. Deletions in genes encoding ESD, UCHL3 and ABHD13 were observed in more than $38 \%$ of the samples (Fig. 4a). These results were validated in the MMRF CoMMpass dataset: PNPLA6 was found to have a copy number gain in $40.1 \%(419 / 1044)$ of samples, CEL was found to have a copy number gain in $38.5 \%$ $(402 / 1044)$ of samples and PAFAH1B3 had a copy number gain in $31.7 \%(331 / 1044)$ of samples. Deletions in genes encoding ESD, UCHL3 and $A B H D 13$ were observed in $\sim 36-41 \%$ of samples (Supplementary Fig. S11). Furthermore, in the FIMM cohort, a 

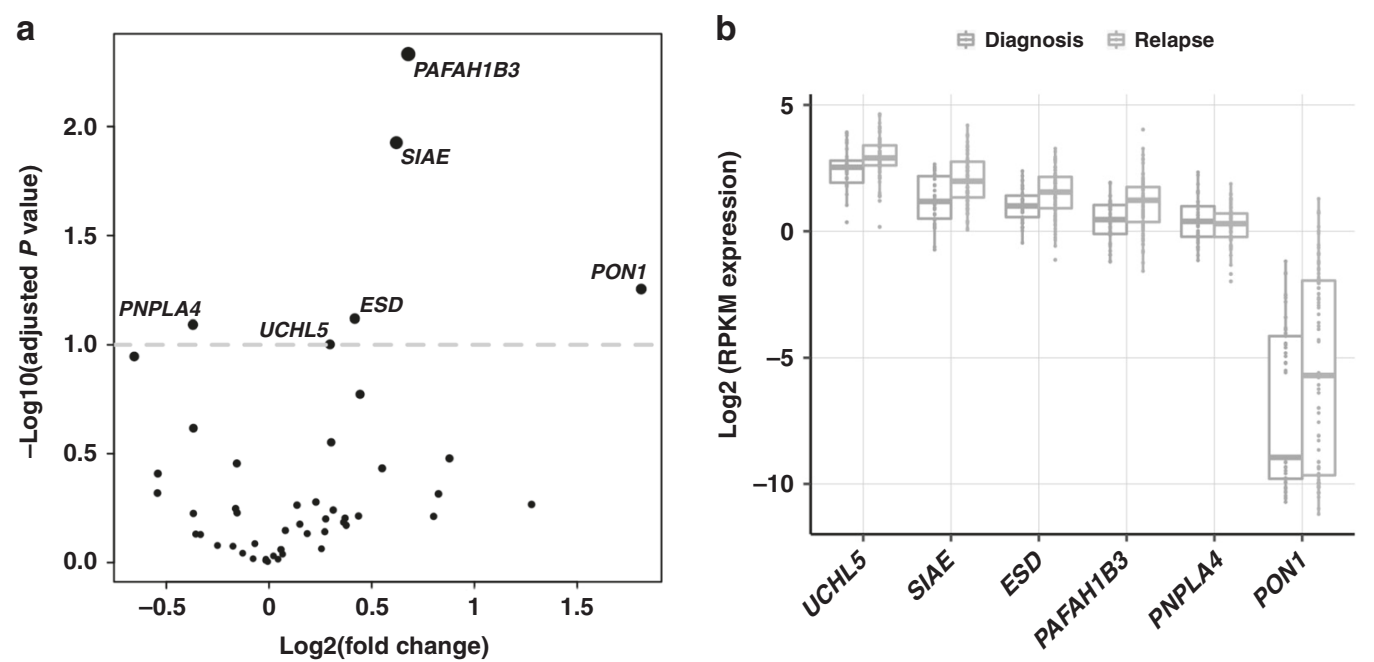

Fig. 2 Esterase genes are differentially expressed in newly diagnosed multiple myeloma $(n=41)$ versus relapsed/refractory multiple myeloma $(\boldsymbol{n}=\mathbf{8 2})$ samples in the in-house FIMM dataset. a DEseq2 differential expression results for 51 esterase genes. $\mathbf{b}$ The expression values of genes $(n=6)$ predicted to be differentially regulated in NDMM versus RRMM groups ${ }^{\mathrm{a}}$. FIMM Institute for Molecular Medicine Finland, NDMM newly diagnosed multiple myeloma, RPKM reads per kilobase of transcript per million mapped reads, RRMM relapsed/refractory multiple myeloma. a Box plots: thick central line represents median; top and bottom lines of box represent third quartile and first quartile; whiskers indicate the variability in the data outside the upper and lower quartile; circles inside the boxplot/distribution represent data point locations; circles outside the boxplot/distribution represent outliers.

correlation analysis of gene expression and CNVs revealed that among all genes predicted to have CNVs, only the genes UCHL5 and UCHL3 had a weak positive correlation $(r>0.5)$ of 0.57 and 0.54 with corresponding log2(RPKM) gene expression values (Fig. 4b).

\section{DISCUSSION}

A detailed understanding of the role of specific metabolic enzymes in the processing of novel therapeutic approaches such as ADCs and PDCs may be critical for their success in the treatment of specific cancers. The majority of chemical linkers in ADCs are based on hydrazone, disulfide, thioester or peptide bonds; these linkers are designed to exploit differences in intracellular $\mathrm{pH}$, intracellular reduction potential or intracellular metabolic enzyme concentrations to break the linker and release a cytotoxic payload into tumour cells. ${ }^{1}$ The cytotoxic activity of PDCs is also dependent on intracellular metabolic enzyme concentrations. $^{2}$ Therefore, among other enzymes, esterases have a significant potential for exploitation in drug design. However, their role in cancer, and more specifically in multiple myeloma, has not been widely studied to date, despite myeloma being a disease of altered protein homoeostasis that is commonly targeted with proteasome inhibitors. Novel lipophilic PDCs such as melflufen (melphalan flufenamide) can readily diffuse into multiple myeloma cells, where high expression of aminopeptidases results in hydrolytic cleavage and rapid release of the cytotoxic alkylator payload, and esterases can also create active intermediate metabolites. ${ }^{3}$ Therefore, the role of these enzymes in myeloma cells is of particular interest; for example, aminopeptidase and esterase gene expression profiles could, hypothetically, be used to identify patient subgroups who are more likely to respond to drugs such as melflufen, which utilise these enzymes as part of their mechanism of action.

In this study, we demonstrate for the first time that several individual esterase genes exhibit relatively high or low median expression in bone marrow aspirates from patients with multiple myeloma. Interestingly, the expression profile of several genes appeared to change on progression from NDMM to RRMM; the expression of UCHL5, sialic acid acetyl esterase (SIAE), esterase D (ESD), PAFAH1B3 and PON1 was significantly higher in RRMM versus NDMM samples, whereas the expression of PNPLA4 was significantly lower in RRMM versus NDMM samples. Among these differentially expressed genes, only UCHL5 was shown to be upregulated in samples with 1q gain (a common cytogenetic abnormality in MM), which is likely due to the genomic location of UCHL5 (1q31.2). UCHL5 is a deubiquitylating enzyme (DUB) that is more highly expressed in MM cells than in normal plasma cells. ${ }^{25}$ Preclinical studies have shown that inhibition of UCHL5 decreases viability and inhibits proliferation of $\mathrm{MM}$ cells, and overcomes resistance to bortezomib. ${ }^{25}$ Many DUBs demonstrate esterase activity, and DUB inhibitors have been studied for anti-myeloma activity. One such inhibitor, VLX1570, has been assessed in a phase 1 study of patients with RRMM; ${ }^{35}$ this study was discontinued due to severe pulmonary toxicity. Nevertheless, further efforts to identify DUB inhibitors with a wider therapeutic index may be warranted given promising preclinical anti-tumour effects and activity in MM resistant to proteasome inhibitors. ${ }^{25,35}$

In our dataset, high expression of OVCA2, PAFAH1B3, SIAE and USP4 was associated with a significantly poorer prognosis compared with samples showing low expression, whereas low expression of GZMA, PCED1B and NXPE3 was associated with a significantly poorer prognosis compared with samples expressing higher levels of the enzyme. Esterase expression patterns were similar in the MMRF CoMMpass validation dataset, but of the genes identified as being prognostic in our dataset, only high expression of PAFAH1B3 and SIAE, and low expression of PCED1B was associated with poor prognosis in the CoMMpass dataset. Other esterases predicted to have a role in disease prognosis only in the validation dataset included NXPE4, UCHL5, PAFAH1B2, BPHL, NXPE1 and UCHL3. The differences observed between datasets may simply have been caused by the lower number of samples in our dataset, or by technical differences in RNA sequencing library preparation methods. Another possible reason for the differences may have been that our dataset includes samples from patients with NDMM $(32.7 \%)$ or RRMM (67.3\%), whereas the CoMMpass dataset includes mostly NDMM samples (87.4\%). Despite these differences, we were still able to identify and validate three prognostic genes.

The other esterases identified as being prognostic in our dataset, but not in the validation dataset, have all previously been reported to be dysregulated in cancer. OVCA2 was originally 
a

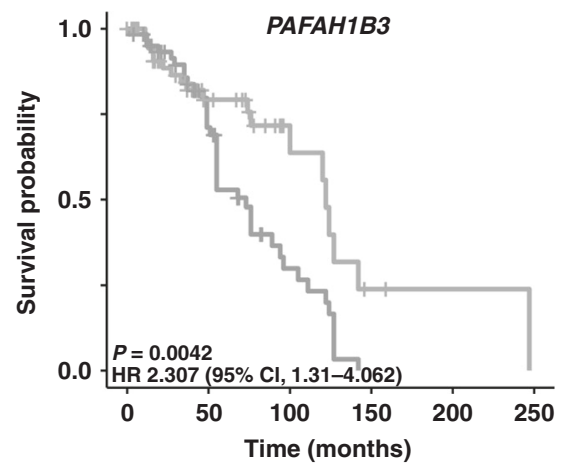

b

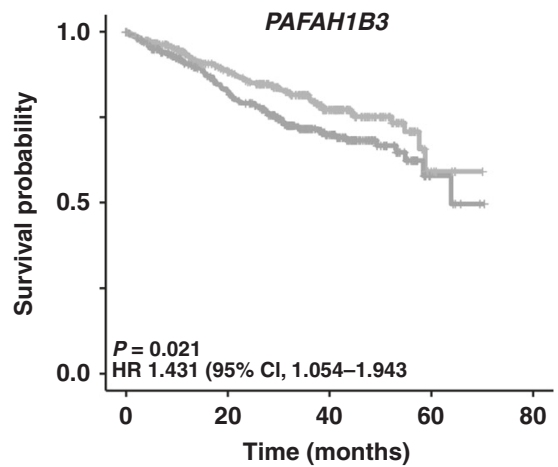

In-house data analysis

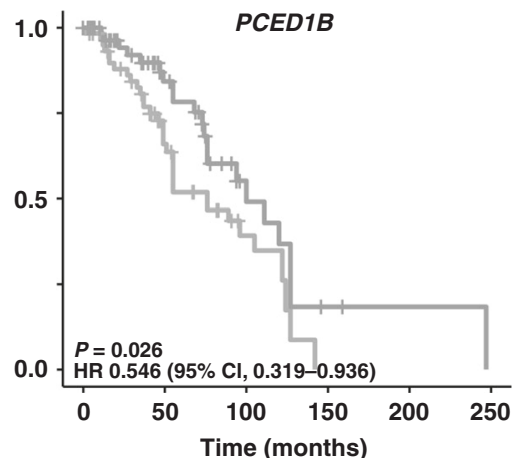

CoMMpass data analysis

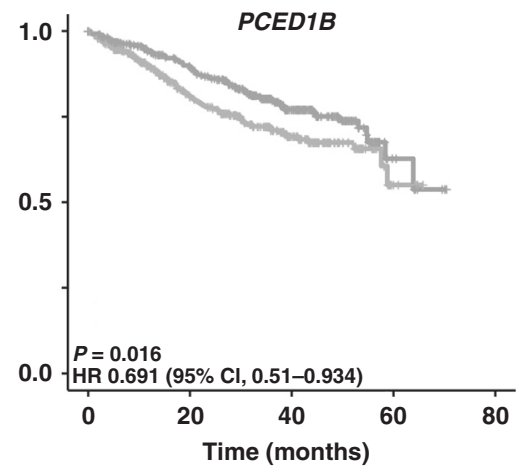

Expression + Low + High
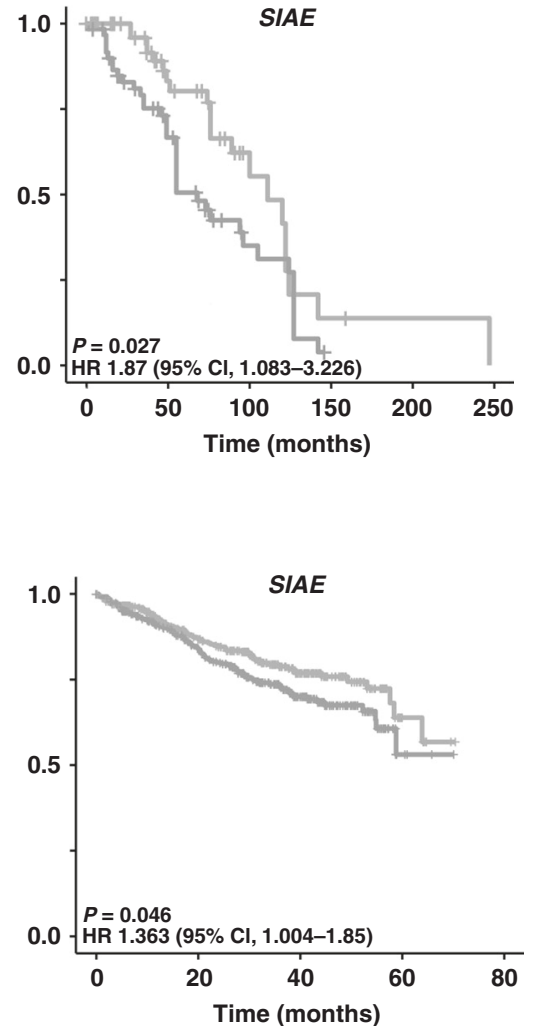

Fig. 3 Prognostic significance of esterase expression. Low expression of PCED1B and high expression of PAFAH1B3 and SIAE are associated with poor prognosis in both (a) the in-house FIMM dataset and (b) the MMRF CoMMpass validation dataset. CL confidence limit, CoMMpass relating clinical outcomes in MM to personal assessment of genetic profile, FIMM Institute for Molecular Medicine Finland, HR hazard ratio, MMRF Multiple Myeloma Research Foundation.

identified as a tumour suppressor gene expressed in healthy surface epithelial cells of the ovary, and is downregulated in the majority of ovarian cell lines and tumours. ${ }^{36}$ OVCA2 is located at chromosome $17 \mathrm{p} 13$, which is commonly deleted in haematological malignancies and also harbours several tumour suppressor genes, including TP53. In multiple myeloma, del17p is widely accepted to be one of the most aggressive features of the disease, and a marker of poor prognosis. ${ }^{36-39}$ In our dataset, OVCA2 was more highly expressed in RRMM versus NDMM samples, whereas in the MMRF CoMMpass dataset there was no difference in expression level. One possible explanation for this difference could be that our dataset had a lower frequency of del17p in RRMM samples $(19 / 82,23.2 \%)$ compared to the MMRF CoMMpass dataset $(14 / 45,31.1 \%)$. Also, in contrast with del17p being a marker of poor prognosis, our analysis of the FIMM dataset suggested that high expression of OVCA2 is associated with poor prognosis in multiple myeloma, despite low expression of OVCA2 being associated with del17p. Further research is required to determine the role of OVCA2 in multiple myeloma. Upregulation of PAFAH1B3 expression in multiple cancers has been observed previously, 4,40,41 with selective inhibition impairing tumour cell survival. ${ }^{42}$ Very little has been published regarding SIAE in cancer cells. A recent in vitro study investigating the potential of targeting the ganglioside GD3 acetylation pathway to treat medulloblastoma showed that SIAE may be associated with mitochondria-mediated apoptosis and etoposide sensitivity. ${ }^{43}$ There are also very few publications on PCED1B. The role of $P C E D 1 B$ antisense RNA was investigated in an in vitro study of glioblastoma, which indicated that it functioned in a hypoxia- inducible factor (HIF)-1-dependent manner and has potential as a prognostic biomarker and druggable target for GBM. ${ }^{44}$ Further investigation into the role of these esterases in multiple myeloma may be warranted.

Our analysis did not take account of gene heterogeneity in CD138+ plasma cells, and the contribution of this heterogeneity to the observed differences in esterase gene expression. Further studies using single-cell RNA sequencing analysis would be useful in this regard. In RRMM patients, it is possible that prior treatment types also influenced the observed esterase gene expression patterns; this is again worthy of further study.

Interestingly, the frequency of SNVs appeared to be rare in the esterase genes, in both our dataset and the MMRF CoMMpass dataset. It has been previously reported that, except for a subset of specific genes, recurrent mutation rates are low in patients with multiple myeloma, suggesting that the dysregulation of key signalling pathways, rather than single-gene mutations, is the key driver for malignancy. ${ }^{18,45}$ Also, multiple myeloma is thought to be genetically heterogeneous, whereby clonal diversity would result in specific mutations only being present in a small number of cells within a tumour, confounding the molecular characterisation of tissue samples. ${ }^{18}$ However, in our study, several esterase genes were found to have a high frequency of duplication and deletion copy number alterations. To explore the possibility that tumours lacking SNVs might be driven by copy number alterations or chromosomal rearrangements, one study reported that of 153 patients with multiple myeloma, $119(77.8 \%)$ had evidence of at least one focal gene copy number gain or loss within a significant peak, including 40 of 60 patients (66.6\%) lacking somatic SNVs in 
a

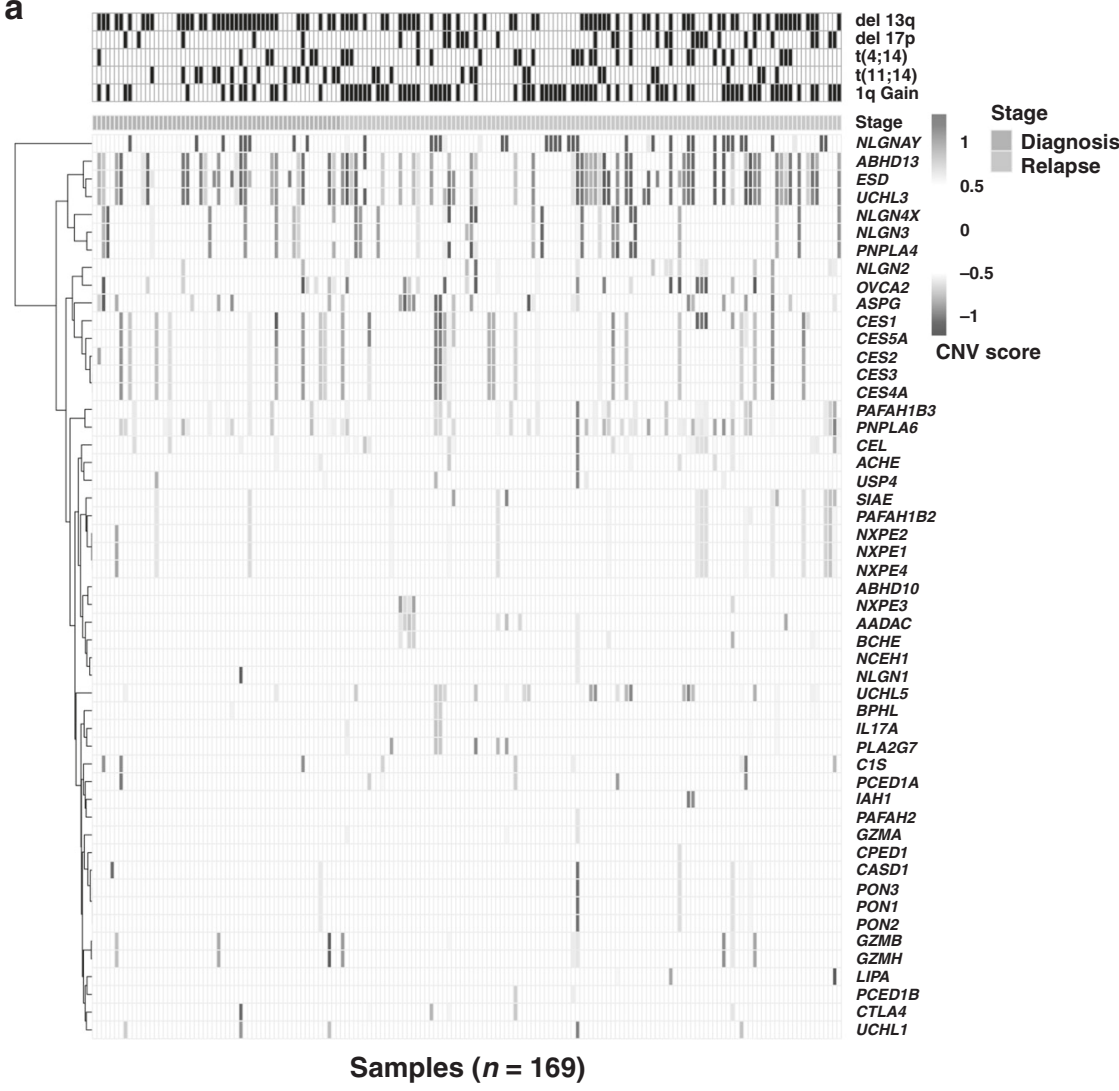

b
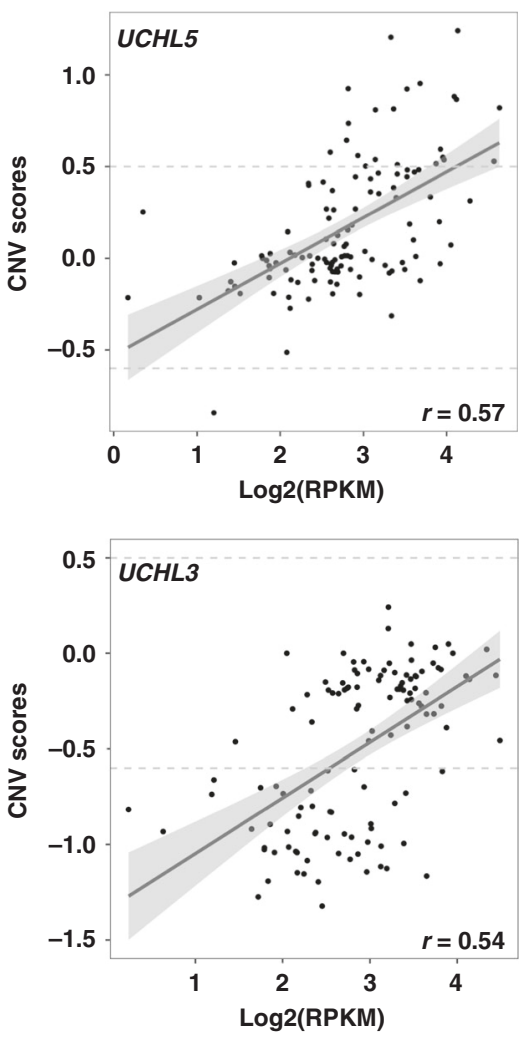

Fig. 4 Multiple esterase genes have copy number alterations. a Heatmap showing clustering of esterase genes based on CNV scores. ${ }^{a}$

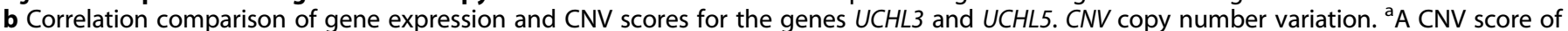
more than 0.5 predicts a duplication/gain event and a CNV score of less than -0.6 predicts a deletion event.

the most significantly mutated genes. ${ }^{18}$ Further studies are required to establish the significance of esterase gene copy number alterations in patients with multiple myeloma.

One limitation of our expression analysis was the sample size; 123 samples were collected from patients with multiple myeloma, preventing statistically robust comparisons with the RRMM or NDMM samples. Another limitation was that matched samples were not widely available for patients progressing from NDMM to RRMM. Finally, regarding verification of the significance of esterase gene expression using the MMRF CoMMpass registry, it should be noted that this dataset was generated from diagnostic samples rather than treatment samples, and that the majority of the samples were taken from patients with NDMM; the possibility that some of our findings are more relevant to RRMM cannot be excluded.

In conclusion, specific esterases exhibited relatively high or low expression in multiple myeloma, and the esterase gene expression profile appeared to change on progression from NDMM to RRMM. High expression of PAFAH1B3 and SIAE, and low expression of $P C E D 1 B$ were identified as poor prognostic markers in patients with multiple myeloma, suggesting a role for these esterases in myeloma biology. Further work is needed to elucidate the biological significance of esterases in cancer, to better understand how they can be effectively utilised to activate anticancer drugs in tumour cells, and their potential applicability as biomarkers of MM and its progression.

\section{ACKNOWLEDGEMENTS}

These data were generated in part by the Multiple Myeloma Research Foundation Personalized Medicine Initiative (https://research.themmrf.org and www.themmrf. org). This work has been supported by funding from Oncopeptides, the iCAN Digital
Precision Cancer Medicine Flagship (Academy of Finland grant 1320185) and the Cancer Society of Finland. We thank Alun Parsons, Minna Suvela, the Institute for Molecular Medicine Finland (FIMM) Sequencing Unit and the FIMM Bioinformatics Unit for their excellent technical support. We are very grateful for the generous donation of samples from patients. Medical writing assistance was provided by Paul Scutt, Spirit Medical Communications Group Limited.

\section{AUTHOR CONTRIBUTIONS}

R.K., N.N.N., F.L. and C.A.H. contributed to the conception/design of the study. All authors contributed to data acquisition, analysis or interpretation; M.M.M., J.L., R.S. and P.A. contributed substantially to data acquisition, and R.K. contributed substantially to data analysis. All authors critically reviewed the drafts, approved the final version and agree to be accountable for all aspects of the work. The corresponding author had full access to the study data and accepts final responsibility for the decision to submit for publication.

\section{ADDITIONAL INFORMATION}

Ethics approval and consent to participate The procedures in this study were approved by an ethical committee of the Helsinki University Hospital Comprehensive Cancer Center (study permit 303/13/03/01/2011) and performed in accordance with the Helsinki Declaration of 1975, as revised in 2008. All patients provided their written informed consent.

\section{Consent to publish Not applicable.}

Data availability All data generated or analysed during this study are included in this article (and its supplementary information files).

Competing interests All authors met the criteria set forth by the International Committee of Medical Journal Editors and hence adequately contributed to the 
manuscript development. J.L. has received personal fees from Amgen, Bristol-Myers Squibb, Celgene, Janssen, Novartis, Pfizer, Roche and Takeda. R.S. has received research funding and personal fees from Amgen, Bristol-Myers Squibb, Celgene and Takeda, and personal fees from Sanofi. N.N.N. is a consultant for Oncopeptides AB. F.L. is an employee of Oncopeptides AB. C.A.H. has received research funding from Celgene, Novartis, Oncopeptides AB, Orion Pharma, Pfizer and the IMI2 consortium project HARMONY. P.A. has received personal fees from Amgen, Bayer, Bristol-Meyers-Squibb, Celgene, GSK, Janssen, Novartis, Sanofi and Takeda. R.K. and M.M.M. have nothing to disclose.

Funding information This work has been supported by funding from Oncopeptides, the iCAN Digital Precision Cancer Medicine Flagship (Academy of Finland grant 1320185), Cancer Society of Finland, and Sigrid Jusélius Foundation. Open Access funding provided by University of Helsinki including Helsinki University Central Hospital.

Supplementary information is available for this paper at https://doi.org/10.1038/ s41416-020-01237-1.

Publisher's note Springer Nature remains neutral with regard to jurisdictional claims in published maps and institutional affiliations.

\section{REFERENCES}

1. Thomas, A., Teicher, B. A. \& Hassan, R. Antibody-drug conjugates for cancer therapy. Lancet Oncol. 17, e254-e262 (2016).

2. Wang, Y., Cheetham, A. G., Angacian, G., Su, H., Xie, L. \& Cui, H. Peptide-drug conjugates as effective prodrug strategies for targeted delivery. Adv. Drug Deliv. Rev. 110-111, 112-126 (2017)

3. Wickstrom, M., Nygren, P., Larsson, R., Harmenberg, J., Lindberg, J., Sjoberg, P. et al. Melflufen-a peptidase-potentiated alkylating agent in clinical trials. Oncotarget 8, 66641-66655 (2017).

4. Kohnz, R. A., Mulvihill, M. M., Chang, J. W., Hsu, K. L., Sorrentino, A., Cravatt, B. F. et al. Activity-based protein profiling of oncogene-driven changes in metabolism reveals broad dysregulation of PAFAH1B2 and 1B3 in cancer. ACS Chem. Biol. 10 1624-1630 (2015).

5. Fukami, T. \& Yokoi, T. The emerging role of human esterases. Drug Metab. Pharmacokinet. 27, 466-477 (2012).

6. McGoldrick, C. A., Jiang, Y. L., Paromov, V., Brannon, M., Krishnan, K. \& Stone, W. L. Identification of oxidized protein hydrolase as a potential prodrug target in prostate cancer. BMC Cancer 14, 77 (2014)

7. Yamazaki, Y., Furukawa, F., Nishikawa, A., Takahashi, M. \& Oka, S. Histochemical determination of stereoselectivity of esterases in normal pancreas and pancreatic tubular adenocarcinoma of hamsters. Biotech. Histochem 73, 23-31 (1998).

8. Yamazaki, Y., Kageyama, Y. \& Okuno, H. Direct evaluation of stereoselectivity of cancer esterases by polyacrylamide gel electrophoresis coupled with activity staining with chiral naphthyl esters. Anal. Biochem. 231, 295-300 (1995).

9. Yamazaki, Y., Ogawa, Y., Afify, A. S., Kageyama, Y., Okada, T., Okuno, H. et al. Difference between cancer cells and the corresponding normal tissue in view of stereoselective hydrolysis of synthetic esters. Biochim. Biophys. Acta 1243, 300-308 (1995)

10. Marini, B. L., Perissinotti, A. J., Bixby, D. L., Brown, J. \& Burke, P. W. Catalyzing improvements in ALL therapy with asparaginase. Blood Rev. 31, 328-338 (2017).

11. Michelozzi, I. M., Granata, V., De Ponti, G., Alberti, G., Tomasoni, C., Antolini, L. et al. Acute myeloid leukaemia niche regulates response to L-asparaginase. Br. J. Haematol. 186, 420-430 (2019).

12. Chauhan, D., Ray, A., Viktorsson, K., Spira, J., Paba-Prada, C., Munshi, N. et al. In vitro and in vivo antitumor activity of a novel alkylating agent, melphalan-flufenamide, against multiple myeloma cells. Clin. Cancer Res. 19, 3019-3031 (2013).

13. Liu, Y. R., Hu, Y., Zeng, Y., Li, Z. X., Zhang, H. B., Deng, J. L. et al. Neurexophilin and PC-esterase domain family member 4 (NXPE4) and prostate androgen-regulated mucin-like protein 1 (PARM1) as prognostic biomarkers for colorectal cancer. J. Cell Biochem. 120, 18041-18052 (2019).

14. Ma, C., Guo, Y., Zhang, Y., Duo, A., Jia, Y., Liu, C. et al. PAFAH1B2 is a HIF1a target gene and promotes metastasis in pancreatic cancer. Biochem. Biophys. Res. Commun. 501, 654-660 (2018).

15. Xi, H. J., Wu, R. P., Liu, J. J., Zhang, L. J. \& Li, Z. S. Role of acetylcholinesterase in lung cancer. Thorac. Cancer 6, 390-398 (2015).

16. Roufas, C., Chasiotis, D., Makris, A., Efstathiades, C., Dimopoulos, C. \& Zaravinos, A The expression and prognostic impact of immune cytolytic activity-related markers in human malignancies: a comprehensive meta-analysis. Front. Oncol. 8, 27 (2018).
17. Hu, Y., Chen, W. \& Wang, J. Progress in the identification of gene mutations involved in multiple myeloma. OncoTargets Ther. 12, 4075-4080 (2019).

18. Lohr, J. G., Stojanov, P., Carter, S. L., Cruz-Gordillo, P., Lawrence, M. S., Auclair, D. et al. Widespread genetic heterogeneity in multiple myeloma: implications for targeted therapy. Cancer Cell 25, 91-101 (2014).

19. Moreaux, J., Klein, B., Bataille, R., Descamps, G., Maiga, S., Hose, D. et al. A high-risk signature for patients with multiple myeloma established from the molecular classification of human myeloma cell lines. Haematologica 96, 574-582 (2011).

20. Yano, H., Kayukawa, S., lida, S., Nakagawa, C., Oguri, T., Sanda, T. et al. Overexpression of carboxylesterase-2 results in enhanced efficacy of topoisomerase I inhibitor, irinotecan (CPT-11), for multiple myeloma. Cancer Sci. 99, 2309-2314 (2008).

21. Kassambara, A., Hose, D., Moreaux, J., Reme, T., Torrent, J., Rossi, J. F. et al. Identification of pluripotent and adult stem cell genes unrelated to cell cycle and associated with poor prognosis in multiple myeloma. PLoS ONE 7, e42161 (2012).

22. Wu, S. P., Pfeiffer, R. M., Ahn, I. E., Mailankody, S., Sonneveld, P., van Duin, M. et al. Impact of genes highly correlated with MMSET myeloma on the survival of nonMMSET myeloma patients. Clin. Cancer Res. 22, 4039-4044 (2016).

23. Ellidag, H. Y., Eren, E., Aydin, O., Yildirim, M., Sezer, C. \& Yilmaz, N. Multiple myeloma: relationship to antioxidant stress. Med. Princ. Pr. 23, 18-23 (2013).

24. Wang, X., Mazurkiewicz, M., Hillert, E. K., Olofsson, M. H., Pierrou, S., Hillertz, P. et al. The proteasome deubiquitinase inhibitor VLX1570 shows selectivity for ubiquitin-specific protease-14 and induces apoptosis of multiple myeloma cells. Nat. Sci. Rep. 26, 26979 (2016).

25. Tian, Z., D’Arcy, P., Wang, X., Ray, A., Tai, Y. T., Hu, Y. et al. A novel small molecule inhibitor of deubiquitylating enzyme USP14 and UCHL5 induces apoptosis in multiple myeloma and overcomes bortezomib resistance. Blood 123, 706-716 (2014).

26. Dobin, A., Davis, C. A., Schlesinger, F., Drenkow, J., Zaleski, C., Jha, S. et al. STAR: ultrafast universal RNA-seq aligner. Bioinformatics 29, 15-21 (2013).

27. Cunningham, F., Achuthan, P., Akanni, W., Allen, J., Amode, M. R., Armean, I. M et al. Ensembl 2019. Nucleic Acids Res. 47, D745-D751 (2019).

28. Ncbi Resource Coordinators. Database resources of the National Center for Biotechnology Information. Nucleic Acids Res. 46, D8-D13 (2018).

29. Love, M. I., Huber, W. \& Anders, S. Moderated estimation of fold change and dispersion for RNA-seq data with DESeq2. Genome Biol. 15, 550 (2014).

30. Koboldt, D. C., Zhang, Q., Larson, D. E., Shen, D., McLellan, M. D., Lin, L. et al. VarScan 2: somatic mutation and copy number alteration discovery in cancer by exome sequencing. Genome Res. 22, 568-576 (2012).

31. Cingolani, P., Platts, A., Wang le, L., Coon, M., Nguyen, T., Wang, L. et al. A program for annotating and predicting the effects of single nucleotide polymorphisms, SnpEff: SNPs in the genome of Drosophila melanogaster strain w1118; iso-2; iso-3. Fly 6, 80-92 (2012).

32. Eldfors, S., Kuusanmaki, H., Kontro, M., Majumder, M. M., Parsons, A., Edgren, H. et al. Idelalisib sensitivity and mechanisms of disease progression in relapsed TCF3-PBX1 acute lymphoblastic leukemia. Leukemia 31, 51-57 (2017).

33. Majumder, M. M., Silvennoinen, R., Anttila, P., Tamborero, D., Eldfors, S., Yadav, B. et al. Identification of precision treatment strategies for relapsed/refractory multiple myeloma by functional drug sensitivity testing. Oncotarget $\mathbf{8}$, 56338-56350 (2017).

34. Ross, F. M., Avet-Loiseau, H., Ameye, G., Gutierrez, N. C., Liebisch, P., O'Connor, S et al. Report from the European Myeloma Network on interphase FISH in multiple myeloma and related disorders. Haematologica 97, 1272-1277 (2012).

35. Rowinsky, E. K., Paner, A., Berdeja, J. G., Paba-Prada, C., Venugopal, P., Porkka, K. et al. Phase 1 study of the protein deubiquitinase inhibitor VLX1570 in patients with relapsed and/or refractory multiple myeloma. Investig. N. Drugs 38, 1448-1453 (2020).

36. Schultz, D. C., Vanderveer, L., Berman, D. B., Hamilton, T. C., Wong, A. J. \& Godwin A. K. Identification of two candidate tumor suppressor genes on chromosome 17p13.3. Cancer Res. 56, 1997-2002 (1996).

37. Thakurta, A., Ortiz, M., Blecua, P., Towfic, F., Corre, J., Serbina, N. V. et al. High subclonal fraction of $17 p$ deletion is associated with poor prognosis in multiple myeloma. Blood 133, 1217-1221 (2019).

38. Lakshman, A., Painuly, U., Rajkumar, S. V., Ketterling, R. P., Kapoor, P., Greipp, P. T. et al. Impact of acquired del(17p) in multiple myeloma. Blood Adv. 3, 1930-1938 (2019).

39. Lakshman, A., Painuly, U., Rajkumar, S. V., Ketterling, R. P., Kapoor, P., Greipp, P. T. et al. Natural history of multiple myeloma with de novo del(17p). Blood Cancer J. 9, 32 (2019).

40. Mulvihill, M. M., Benjamin, D. I., Ji, X., Le Scolan, E., Louie, S. M., Shieh, A. et al Metabolic profiling reveals PAFAH1B3 as a critical driver of breast cancer pathogenicity. Chem. Biol. 21, 831-840 (2014).

41. Xu, J., Zang, Y., Cao, S., Lei, D. \& Pan, X. Aberrant expression of PAFAH1B3 associates with poor prognosis and affects proliferation and aggressiveness in 
hypopharyngeal squamous cell carcinoma. OncoTargets Ther. 12, 2799-2808 (2019).

42. Chang, J. W., Zuhl, A. M., Speers, A. E., Niessen, S., Brown, S. J., Mulvihill, M. M. et al. Selective inhibitor of platelet-activating factor acetylhydrolases $1 \mathrm{~b} 2$ and $1 \mathrm{~b} 3$ that impairs cancer cell survival. ACS Chem. Biol. 10, 925-932 (2015).

43. Mather, R. L., Loveson, K. F. \& Fillmore, H. L. Human sialic acid O-acetyl esterase (SIAE)-mediated changes in sensitivity to etoposide in a medulloblastoma cell line. Sci. Rep. 9, 8609 (2019).

44. Yao, Z., Zhang, Q., Guo, F., Guo, S., Yang, B., Liu, B. et al. Long noncoding RNA PCED1B-AS1 promotes the Warburg effect and tumorigenesis by upregulating HIF-1a in glioblastoma. Cell Transplant. 29, 963689720906777 (2020).

45. Walker, B. A., Boyle, E. M., Wardell, C. P., Murison, A., Begum, D. B., Dahir, N. M. et al. Mutational spectrum, copy number changes, and outcome: results of a sequencing study of patients with newly diagnosed myeloma. J. Clin. Oncol. 33, 3911-3920 (2015).
Open Access This article is licensed under a Creative Commons Attribution 4.0 International License, which permits use, sharing, adaptation, distribution and reproduction in any medium or format, as long as you give appropriate credit to the original author(s) and the source, provide a link to the Creative Commons license, and indicate if changes were made. The images or other third party material in this article are included in the article's Creative Commons license, unless indicated otherwise in a credit line to the material. If material is not included in the article's Creative Commons license and your intended use is not permitted by statutory regulation or exceeds the permitted use, you will need to obtain permission directly from the copyright holder. To view a copy of this license, visit http://creativecommons. org/licenses/by/4.0/.

C The Author(s) 2021 